\title{
Effects of transverse momentum broadening of parton cascades from coherent emissions and scatterings in a medium
}

\author{
E. Blanco, ${ }^{a}$ K. Kutak, ${ }^{a}$ M. Rohrmoser, ${ }^{a, *}$ W. Płaczek ${ }^{b}$ and R. Straka ${ }^{c}$ \\ ${ }^{a}$ Institute of Nuclear Physics, Polish Academy of Sciences, \\ ul. Radzikowskiego 152, 31-342 Kraków, Poland \\ ${ }^{b}$ Institute of Applied Computer Science, Jagiellonian University, \\ ul. Łojasiewicza 11, 30-348 Kraków, Poland \\ ${ }^{c}$ AGH University of Science and Technology, \\ Kraków, Poland \\ E-mail: martin.rohrmoser@ifj.edu.pl, eti.bla@orange.fr, \\ krzysztof.kutak@ifj.edu.pl, Wieslaw.Placzek@uj.edu.pl, strakrob@gmail.com
}

Highly energetic particles in the medium of a quark gluon plasma undergo processes of coherent medium induced radiation and scatterings off medium particles. We solved the evolution equations for particle fragmentation functions that describe in-medium evolution via these two in-medium processes numerically via Monte-Carlo algorithms. We study the in-medium broadening of the distribution over momentum components transverse to the jet-axis for different cases of jet-medium interactions and different values of average transverse momentum transfer.

\footnotetext{
*** Particles and Nuclei International Conference - PANIC2021 ***

*** 5 - 10 September, $2021 * * *$

$* * *$ Online $* * *$
}

\footnotetext{
${ }^{*}$ Speaker
} 
In this work, we particularly focus on the evolution of parton jets, highly energetic collimated sprays of strongly interacting particles, which can be used as a means to study the hot and dense medium of a quark gluon plasma (QGP). Within the medium jet particles can undergo processes of scatterings off medium particles. These scattering processes can also involve particle momenta off the mass shell, which lead to emissions of new particles. The emissions can occur simultaneously to multiple scatterings off medium particles giving rise to interference effects. The spectra for these coherent medium induced radiations were first described for a QCD medium by Baier, Dokshitzer, Mueller, Peigné, Schiff and independently by Zakharov (BDMPS-Z) [1-7]. Later on an effective splitting kernel for coherent medium induced radiations that also allows to reproduce the BDMPS-Z emission spectra was obtained in [8] as

$$
\mathcal{K}\left(\mathbf{Q}, z, p_{0}^{+}\right)=\frac{2}{p_{0}^{+}} \frac{P_{g g}(z)}{z(1-z)} \sin \left[\frac{\mathbf{Q}^{2}}{2 \omega_{0} \hat{q}_{0}}\right] \exp \left[-\frac{\mathbf{Q}^{2}}{2 \omega_{0} \hat{q}_{0}}\right]
$$

with

$$
\mathbf{Q}=\mathbf{k}-z \mathbf{q}, \omega_{0}=z(1-z) p_{0}^{+}, \quad \hat{q}_{0}=\hat{q}(1-z(1-z)), \quad P_{g g}(z)=N_{c} \frac{[1-z(1-z)]^{2}}{z(1-z)},
$$

where $p_{0}^{+}=E$ is the energy of the initial jet particle, $z$ the parton momentum fraction (with regard to the decaying particle), $\mathbf{k}$ the jet-particle momentum components transverse to the jet axis, $\hat{q}$ the average transverse momentum transfer, $\alpha_{S}$ the QCD coupling constant and $N_{C}$ the number of colors. An evolution equation for the fragmentation function $D(x, \mathbf{k}, t)$ of gluons that includes besides coherent medium induced radiations also scatterings has been formulated $[9,10]$ as

$$
\begin{aligned}
\frac{\partial}{\partial t} D(x, \mathbf{k}, t) & =\alpha_{s} \int_{0}^{1} d z \int \frac{d^{2} q}{(2 \pi)^{2}}\left[2 \mathcal{K}\left(\mathbf{Q}, z, \frac{x}{z} p_{0}^{+}\right) D\left(\frac{x}{z}, \mathbf{q}, t\right)-\mathcal{K}\left(\mathbf{q}, z, x p_{0}^{+}\right) D(x, \mathbf{k}, t)\right] \\
& +\int \frac{d^{2} \mathbf{l}}{(2 \pi)^{2}} w(\mathbf{l}) D(x, \mathbf{k}-\mathbf{l}, t)-\int \frac{d^{2} \mathbf{I}^{\prime}}{(2 \pi)^{2}} w\left(\mathbf{I}^{\prime}\right) D(x, \mathbf{k}, t) .
\end{aligned}
$$

For our current work we used the following scattering kernels from [9, 10] and [11]

$$
\begin{aligned}
& w(\mathbf{l})=\frac{16 \pi^{2} \alpha_{s}^{2} N_{c} n}{\mathbf{l}^{4}}, \quad \text { and } \\
& w(\mathbf{l})=\frac{4 \pi \alpha_{s} m_{D}^{2} T}{\mathbf{l}^{2}\left(\mathbf{l}^{2}+m_{D}^{2}\right)} .
\end{aligned}
$$

where $n$ is the density of scatterers in the medium and $m_{D}$ the Debye mass.

Via integration over the transverse momentum components an effective collinear splitting kernel $\mathcal{K}(z)$ can be obtained as

$$
\mathcal{K}(z)=\int d^{2} \mathbf{Q} \mathcal{K}\left(\mathbf{Q}, z, y p_{0}^{+}\right) \frac{\sqrt{y}}{2 \pi} t^{*} \frac{\alpha_{s} N_{c}}{\pi}=\frac{(1-z(1-z))^{5 / 2}}{(z(1-z))^{3 / 2}}, \quad \text { with } t^{*}=\frac{\pi}{\alpha_{s} N_{c}} \sqrt{\frac{p_{0}^{+}}{\hat{q}}} .
$$

which corresponds to the following evolution equation $[9,10]$

$$
\begin{aligned}
\frac{\partial}{\partial t} D(x, \mathbf{k}, t) & =\frac{1}{t^{*}} \int_{0}^{1} d z \mathcal{K}(z)\left[\frac{1}{z^{2}} \sqrt{\frac{z}{x}} D\left(\frac{x}{z}, \frac{\mathbf{k}}{z}, t\right) \theta(z-x)-\frac{z}{\sqrt{x}} D(x, \mathbf{k}, t)\right] \\
& +\int \frac{d^{2} \mathbf{l}}{(2 \pi)^{2}} w(\mathbf{l}) D(x, \mathbf{k}-\mathbf{l}, t)-\int \frac{d^{2} \mathbf{l}^{\prime}}{(2 \pi)^{2}} w\left(\mathbf{l}^{\prime}\right) D(x, \mathbf{k}, t) .
\end{aligned}
$$


After integration over the transverse momentum an evolution equation for the fragmentation functions integrated over transverse momentum $D(x, t)$ can be obtained as

$$
\frac{\partial}{\partial t} D(x, t)=\frac{1}{t^{*}} \int_{0}^{1} d z \mathcal{K}(z)\left[\sqrt{\frac{z}{x}} D\left(\frac{x}{z}, t\right) \theta(z-x)-\frac{z}{\sqrt{x}} D(x, t)\right] .
$$

For the here presented work, we solved the evolution equations (3), (7), and (8) numerically via Monte-Carlo algorithms $[12,13]$. The following six cases of in-medium jet-evolution were studied: three cases with non-collinear branching, which follow Eq. (3), where the scattering kernels either follow Eq. (4), Eq. (5) or $w(q)=0$, two cases with collinear branching that follow Eq. (3), where the scattering kernels either follow Eq. (4), Eq. (5), and a case that will be referred to as Gaussian approximation. In the Gaussian approximation $D(x, \mathbf{k}, t)$ is given by

$$
D(x, \mathbf{k}, t)=D(x, t) \frac{4 \pi}{\left\langle k_{\perp}^{2}\right\rangle} \exp \left[-\frac{\mathbf{k}^{2}}{\left\langle k_{\perp}^{2}\right\rangle}\right], \quad \text { with }\left\langle k_{\perp}^{2}\right\rangle=\min \left\{\frac{1}{2} \hat{q} t\left(1+x^{2}\right), \frac{x E \hat{q}}{4 \bar{\alpha}},(x E)^{2}\right\},
$$

where $D(x, t)$ follows Eq. (8) and it is assumed that $k_{\perp}^{2}<\omega^{2}=(x E)^{2}$. These models were used to study the relative importance of non-collinear branching and scattering for transverse momentum broadening. For the numerical calculations a few constant parameters were assumed as well as a medium described by constant parameters. These parameters are

$$
\hat{q}=1 \mathrm{GeV}^{2} / \mathrm{fm}, \alpha_{s}=\frac{\pi}{10}, n=0.243 \mathrm{GeV}^{3}, m_{D}=0.993 \mathrm{GeV}, p_{0}^{+}=100 \mathrm{GeV}, t=4 \mathrm{fm} / \mathrm{c},
$$

where $t$ is the time that the jets need to traverse the medium. We studied the dependence of the distribution $\tilde{D}\left(x, k_{T}, t\right)=\int_{0}^{2 \pi} d \phi k_{T} D(x, \mathbf{k}, t)$, with $k_{T}=\|\mathbf{k}\|$ on varying values of the average transverse momentum transfer $\hat{q}$. Results are shown in Fig. 1. It can be seen that for the Gaussian model and the model with non-collinear branching without scattering the distributions in transverse momentum become broader for increasing values of $\hat{q}$. However, for cases with both branching as well as scattering the distributions for the smallest $\hat{q}$ value, $\hat{q}=0.5 \mathrm{GeV}^{3} / \mathrm{fm}$, is the broadest, indicating a non-trivial dependence on the interplay between broadenings and scatterings.

\section{Acknowledgments}

This work was partially supported by the Polish National Science Centre with the grant no. DEC-2017/27/B/ST2/01985.

\section{References}

[1] R. Baier, D. Schiff and B.G. Zakharov, Energy loss in perturbative QCD, Ann. Rev. Nucl. Part. Sci. 50 (2000) 37 [hep-ph/0002198].

[2] R. Baier, A.H. Mueller, D. Schiff and D.T. Son, 'Bottom up' thermalization in heavy ion collisions, Phys. Lett. B 502 (2001) 51 [hep-ph/0009237].

[3] B.G. Zakharov, Fully quantum treatment of the Landau-Pomeranchuk-Migdal effect in QED and QCD, JETP Lett. 63 (1996) 952 [hep-ph/9607440]. 


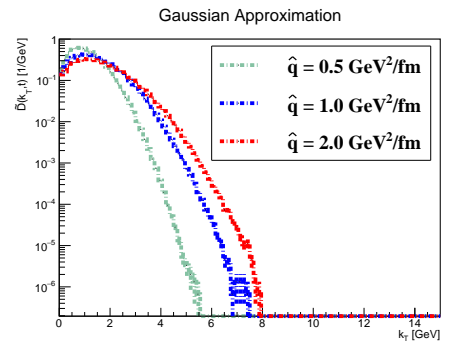

$\mathrm{K}(\mathrm{Q}, \mathrm{z}), \mathrm{W}(\mathrm{I})=0$

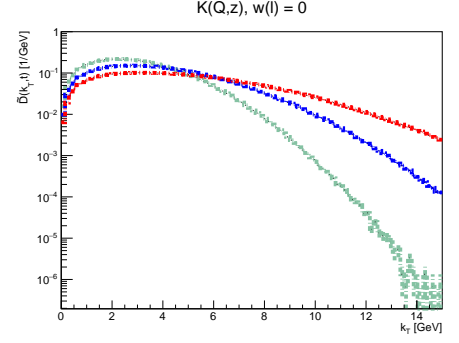

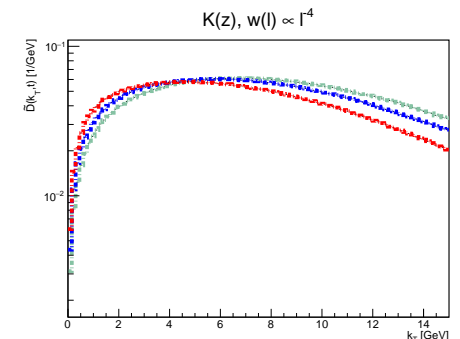

$K(Q, z), w(l) \propto r^{4}$

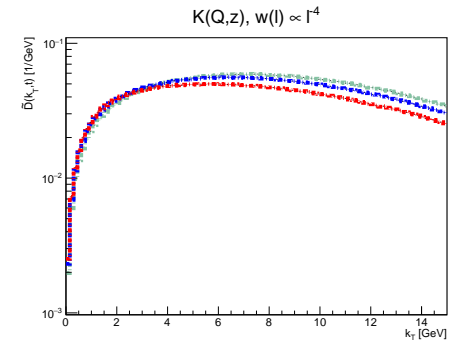

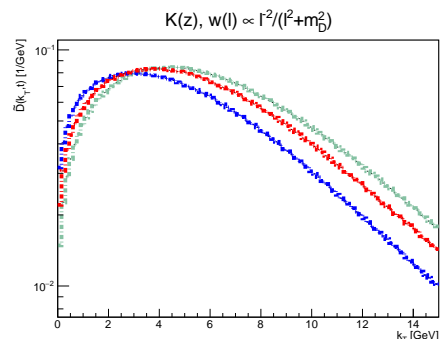

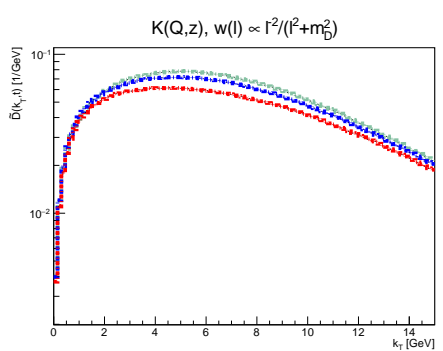

Figure 1: Transverse momentum distributions for varying values of $\hat{q}$ in different models as indicated.

[4] B.G. Zakharov, Radiative energy loss of high-energy quarks in finite size nuclear matter and quark - gluon plasma, JETP Lett. 65 (1997) 615 [hep-ph/9704255].

[5] B.G. Zakharov, Transverse spectra of radiation processes in-medium, JETP Lett. 70 (1999) 176 [hep-ph/9906536].

[6] R. Baier, Y.L. Dokshitzer, S. Peigne and D. Schiff, Induced gluon radiation in a QCD medium, Phys. Lett. B 345 (1995) 277 [hep-ph/9411409].

[7] R. Baier, Y.L. Dokshitzer, A.H. Mueller, S. Peigne and D. Schiff, The Landau-Pomeranchuk-Migdal effect in QED, Nucl. Phys. B 478 (1996) 577 [hep-ph/9604327].

[8] J.-P. Blaizot, F. Dominguez, E. Iancu and Y. Mehtar-Tani, Medium-induced gluon branching, JHEP 01 (2013) 143 [1209.4585].

[9] J.-P. Blaizot, F. Dominguez, E. Iancu and Y. Mehtar-Tani, Probabilistic picture for medium-induced jet evolution, JHEP 06 (2014) 075 [1311. 5823].

[10] J.-P. Blaizot, L. Fister and Y. Mehtar-Tani, Angular distribution of medium-induced QCD cascades, Nucl. Phys. A940 (2015) 67 [1409.6202].

[11] P. Aurenche, F. Gelis and H. Zaraket, A Simple sum rule for the thermal gluon spectral function and applications, JHEP 05 (2002) 043 [hep-ph/0204146].

[12] K. Kutak, W. Płaczek and R. Straka, Solutions of evolution equations for medium-induced QCD cascades, Eur. Phys. J. C 79 (2019) 317 [1811.06390].

[13] E. Blanco, K. Kutak, W. Płaczek, M. Rohrmoser and R. Straka, Medium induced QCD cascades: broadening and rescattering during branching, JHEP 04 (2021) 014 [2009.03876]. 